\section{À propos de l'utilisation du propofol pour la mobilisation des patients atteints de fracture de la banche [Propofol to facilitate mobilization of patients with a hip fracture]}

Au rédacteur en chef, Je voudrais commenter sur l'article de Minville et coll. ${ }^{1}$ paru récemment dans le Journal canadien d'anesthésie. Les auteurs ont montré de bons résultats sur l'efficacité du propofol pour permettre la mobilisation des patients âgés atteints d'une fracture du fémur en vue de réaliser une rachianesthésie, mais quelques considérations doivent être faites. La chirurgie de la fracture de la hanche se fait souvent chez des patients avec des maladies chroniques, notamment des maladies cardiaques et vasculaires, de l'anémie (chronique et aiguë), de l'hypertension, et de la déshydratation. La rachianesthésie produit un bloc nerveux sympathique, avec vasodilatation et hypotension artérielle secondaire, qui s'ajoute aux conditions médicales de base. Cette situation est similaire du point de vue pathophysiologique au choc hypovolémique hémorragique aigu. Si l'on choisit le propofol pour la sédation profonde avant l'induction de l'anesthésie, il est possible que l'hypotension et la dépression myocardique prolongées que ce médicament produit ${ }^{2,3}$ aggravent les effets hémodynamiques provoqués par le bloc neuraxial. Malheureusement nous ne pouvons pas connaitre cette influence parce que les données hémodynamiques intraopératoires immédiates et plus tardives, ainsi que le besoin d'éphédrine ne sont pas montrés dans l'étude.

Par ailleurs, les auteurs de l'étude ne citent qu'un seul article sur l'analgésie préopératoire dans les cas de fracture de fémur, mais une recherche superficielle avec Medline-PubMed (www.ncbi.nlm.nih.gov/ entrez/) employant les mots clés " anesthesia and hip fracture ", avec la limitation de date '2000 à 2007' a montré au moins 11 articles (de 139 initialement retrouvés).

Finalement, je suis d'accord avec l'affirmation des auteurs que l'anesthésie locorégionale, y compris la rachianesthésie, est préférable pour ces patients, ${ }^{4}$ et que l'administration d'un analgésique doit être assurée pour le confort du malade lors de la mobilisation et du positionnement pour l'anesthésie. Cet objectif peut être atteint chez ces patients âgés avec de faibles doses de fentanyl $(15-25 \mu \mathrm{g})$, de midazolam ( $1-2$ $\mathrm{mg}$ ), de kétamine (10-25 mg) ou de propofol (10-20 $\mathrm{mg}$ ), administrées seules ou en combinaison selon les besoins. La récupération des effets hypnotiques de ces médicaments est aussi rapide.

Carlos L. Errando MD PhD

Consorcio Hospital General Universitario de

Valencia, Valencia, Espagne

Courriel : c.l.errando@carloserrando.com

Conflits d'intérêt et sources de financement : Aucun. Accepté pour publication le 28 mai 2007.

\section{Références}

1 Minville V, Castel A, Asehnoune K, et al. Le propofol pour réaliser une rachianesthésie en position latérale chez les victimes d'une fracture du fémur. Can J Anesth 2006; 53: 1186-9.

2 Shafer SL. Shock values. Anesthesiology 2004; 101: 567-8.

3 Johnson KB, Egan TD, Kern SE, McJames SW, Cluff $M L$, Pace NL. Influence of hemorrhagic shock followed by crystalloid resuscitation on propofol: a pharmacokinetic and pharmacodynamic analysis. Anesthesiology 2004; 101: 647-59.

4 Beaupre LA, Jones CA, Saunders LD, Jobnston DW, Buckingham J, Majumdar SR. Best practices for elderly hip fracture patients. A systematic overview of the evidence. J Gen Intern Med 2005; 20: 1019-25.

\section{Réponse:}

Tout d'abord, même si la littérature prouve qu'il faut opérer rapidement ces patients, il faut prendre le temps de les optimiser: l'anémie et l'hypovolémie doivent être corrigées avant l'intervention. ${ }^{\text {l }}$

Ensuite, dans notre étude l'utilisation de très faibles

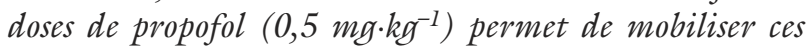
patients fragiles sans aucun retentissement hémodynamique. ${ }^{2}$ Le temps d'ôter la traction, de positionner confortablement le patient, de faire un lavage des mains chirurgical (geste réalisé en casaque stérile), de préparer l'asepsie de la zone de ponction (lavage en quatre temps), d'installer le matériel nécessaire à la réalisation de la rachianesthésie, et de prendre les repères, le patient est totalement réveillé. Il n'y a donc pas de retentissement hémodynamique pendant la mobilisation, ${ }^{2}$ mais encore moins lors de la réalisation de la rachianesthésie. De plus, nous choisissons une rachianesthésie continue, ce qui limite le retentissement hémodynamique lié au bloc sympathique. ${ }^{3}$ Les données hémodynamiques peropératoires sortaient $d u$ cadre $d u$ protocole; cependant, il n'y a pas eu d'hypotension peropératoire. Nous sommes donc loin du modèle expérimental de choc hémorragique suggéré par le Dr Errando. 